\title{
Water in massive star-forming regions: HIFI observations of W3 IRS5 ${ }^{\star}$
}

\author{
L. Chavarría ${ }^{1}$, F. Herpin ${ }^{1}$, T. Jacq ${ }^{1}$, J. Braine ${ }^{1}$, S. Bontemps ${ }^{1}$, A. Baudry ${ }^{1}$, M. Marseille ${ }^{2}$, F. van der Tak Ta $^{2,3}$, \\ B. Pietropaoli1 ${ }^{1,4}$, F. Wyrowski ${ }^{5}$, R. Shipman ${ }^{2}$, W. Frieswijk ${ }^{2}$, E. F. van Dishoeck ${ }^{6,7}$, J. Cernicharo ${ }^{8}$, R. Bachiller ${ }^{9}$, \\ M. Benedettini1 ${ }^{10}$, A. O. Benz ${ }^{11}$, E. Bergin ${ }^{12}$, P. Bjerkeli ${ }^{13}$, G. A. Blake ${ }^{14}$, S. Bruderer ${ }^{11}$, P. Caselli ${ }^{15,16}$, C. Codella ${ }^{16}$, \\ F. Daniel ${ }^{8}$, A. M. di Giorgio ${ }^{10}$, C. Dominik ${ }^{17,18}$, S. D. Doty ${ }^{19}$, P. Encrenaz ${ }^{20}$, M. Fich ${ }^{21}$, A. Fuente ${ }^{22}$, T. Giannini2 ${ }^{23}$, \\ J. R. Goicoechea ${ }^{8}$, Th. de Graauw ${ }^{2}$, P. Hartogh ${ }^{24}$, F. Helmich ${ }^{2}$, G. J. Herczeg 7 , M. R. Hogerheijde ${ }^{6}$, D. Johnstone ${ }^{25,26}$, \\ J. K. Jørgensen ${ }^{27}$, L. E. Kristensen ${ }^{6}$, B. Larsson ${ }^{28}$, D. Lis ${ }^{29}$, R. Liseau ${ }^{13}$, C. M Coey $^{21,30}$, G. Melnick ${ }^{31}$, B. Nisini ${ }^{23}$,' \\ M. Olberg ${ }^{13}$, B. Parise ${ }^{5}$, J. C. Pearson ${ }^{32}$, R. Plume ${ }^{33}$, C. Risacher ${ }^{2}$, J. Santiago-García ${ }^{34}$, P. Saraceno ${ }^{10}$, J. Stutzki ${ }^{35}$, \\ R. Szczerba ${ }^{36}$, M. Tafalla ${ }^{9}$, A. Tielens ${ }^{6}$, T. A. van Kempen ${ }^{31}$, R. Visser ${ }^{6}$, S. F. Wampfler ${ }^{11}$, \\ J. Willem ${ }^{2}$, and U. A. Yildiz \\ (Affiliations are available on page 5 of the online edition)
}

Received 31 May 2010 / Accepted 20 July 2010

\section{ABSTRACT}

\begin{abstract}
We present Herschel observations of the water molecule in the massive star-forming region W3 IRS5. The o- $\mathrm{H}_{2}^{17} \mathrm{O} 1_{10}-1_{01}, \mathrm{p}-\mathrm{H}_{2}^{18} \mathrm{O} 1_{11}-\mathrm{O}_{00}, \mathrm{p}-\mathrm{H}_{2} \mathrm{O}$ $2_{02}-1_{11}, \mathrm{p}-\mathrm{H}_{2} \mathrm{O} 1_{11}-0_{00}, \mathrm{o}-\mathrm{H}_{2} \mathrm{O} 2_{21}-2_{12}$, and o- $\mathrm{H}_{2} \mathrm{O} 2_{12}-1_{01}$ lines, covering a frequency range from 552 up to $1669 \mathrm{GHz}$, have been detected at high spectral resolution with HIFI. The water lines in W3 IRS5 show well-defined high-velocity wings that indicate a clear contribution by outflows. Moreover, the systematically blue-shifted absorption in the $\mathrm{H}_{2} \mathrm{O}$ lines suggests expansion, presumably driven by the outflow. No infall signatures are detected. The $\mathrm{p}-\mathrm{H}_{2} \mathrm{O} 1_{11}-0_{00}$ and o- $\mathrm{H}_{2} \mathrm{O} 2_{12}-1_{01}$ lines show absorption from the cold material $(T \sim 10 \mathrm{~K})$ in which the high-mass protostellar envelope is embedded. One-dimensional radiative transfer models are used to estimate water abundances and to further study the kinematics of the region. We show that the emission in the rare isotopologues comes directly from the inner parts of the envelope $(T \gtrsim 100 \mathrm{~K})$ where water ices in the dust mantles evaporate and the gas-phase abundance increases. The resulting jump in the water abundance (with a constant inner abundance of $10^{-4}$ ) is needed to reproduce the $\mathrm{o}-\mathrm{H}_{2}^{17} \mathrm{O} 1_{10}-1_{01}$ and $\mathrm{p}-\mathrm{H}_{2}^{18} \mathrm{O} 1_{11}-0_{00}$ spectra in our models. We estimate water abundances of $10^{-8}$ to $10^{-9}$ in the outer parts of the envelope $(T \lesssim 100 \mathrm{~K})$. The possibility of two protostellar objects contributing to the emission is discussed.
\end{abstract}

Key words. stars: formation - stars: massive - ISM: molecules - ISM: abundances - dust, extinction - radio lines: ISM

\section{Introduction}

The water molecule is a key species for studying star formation. In the cool regions of molecular clouds $(T<100 \mathrm{~K})$, water is present as ice in the mantles of dust grains. In the immediate surroundings of high-mass protostars, the dust is heated to temperatures well above $100 \mathrm{~K}$, evaporating the water ices and increasing its abundance in the gas phase by several orders of magnitude and making water one of the most abundant molecules. Understanding how the accretion of matter overcomes radiative pressure in massive protostars is a major astrophysical problem (Zinnecker \& Yorke 2007), and water may play an active role in the energy balance (e.g. Doty \& Neufeld 1997). Due to the abundance jump, water makes it possible to specifically study the inner regions from which the massive protostar accretes.

Low angular-resolution water observations of protostellar envelopes (region $\lesssim 0.1 \mathrm{pc}$ in size with a density $n\left(\mathrm{H}_{2}\right) \gtrsim$ $10^{6} \mathrm{~cm}^{-3}$ and centrally peaked density and temperature profiles) with ISO and the Submillimeter Wave Astronomy Satellite

* Herschel is an ESA space observatory with science instruments provided by European-led Principal Investigator consortia and with important participation from NASA.
(SWAS) have revealed overall abundances of less than $10^{-7}$ (Boonman et al. 2003). The much smaller beam of the Herschel Space Observatory (Pilbratt et al. 2010), together with the high spectral resolution of the Heterodyne Instrument for the FarInfrared (HIFI, de Graauw et al. 2010), gives a unique opportunity to separate the emission and absorption in the inner $(T \gtrsim 100 \mathrm{~K})$ and outer $(T \lesssim 100 \mathrm{~K})$ protostellar envelope in order to build a model of the structure and kinematics of these innermost regions, which are critical to fueling the future star.

In this letter, we report water observations of the region W3 IRS5 (Wynn-Williams et al. 1972). W3 IRS5 is a bright infra-red source located in the active star-forming region W3 in the Perseus arm at a distance of $2.0 \mathrm{kpc}$ (Hachisuka et al. 2006). Its high FIR luminosity $\left(1-2 \times 10^{5} L_{\odot}\right.$, Ladd et al. 1993) and the detection of radio emission (Wilson et al. 2003; van der Tak et al. 2005) indicates that W3 IRS5 hosts high-mass stars in an early stage of evolution. High-resolution near-IR images show a cluster of IR-sources in W3 IRS5 with two sources identified as the main elements (Fig. 1 and Megeath et al. 2005). These correspond to the bright millimeter sources MM1 and MM2 identified by Rodón et al. (2008). They are separated by $\sim 1$ " and drive two of the several outflows identified by Boonman et al. (2003) and Rodón et al. (2008). 


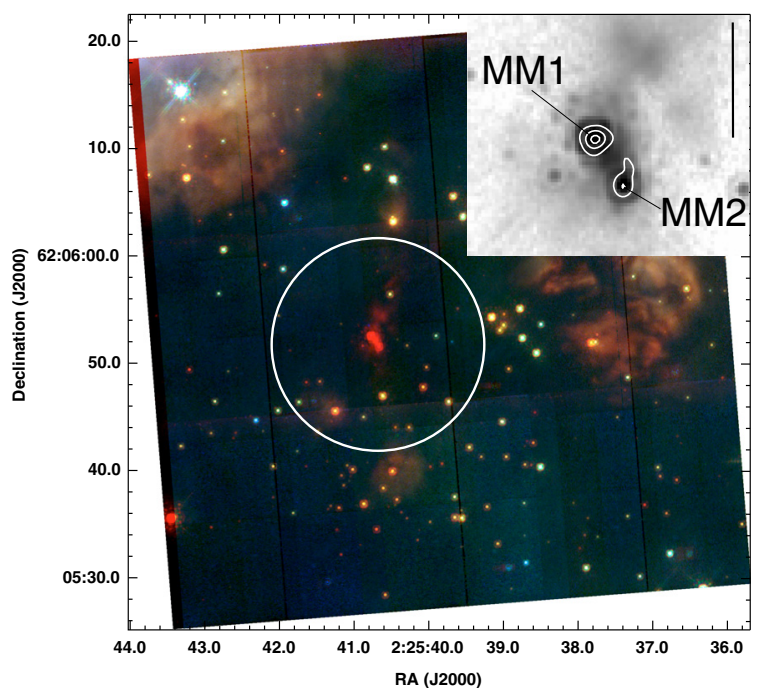

Fig. 1. NICMOS color-composite image of W3 IRS5 and its surroundings from Megeath et al. (2005). The white circle represents the HIFI beam size for the $\mathrm{p}-\mathrm{H}_{2} \mathrm{O} 1_{11}-0_{00}$ and $\mathrm{p}-\mathrm{H}_{2}^{18} \mathrm{O} 1_{11}-0_{00}$ lines $\left(20^{\prime \prime}\right)$. The gray-scale inset is a close-up of the central area at 2.2 microns with white contours corresponding to 30,60 , and $90 \%$ of the peak flux at $1.4 \mathrm{~mm}$ (Rodón et al. 2008). The black bar (top right of inset) represents a distance of $5000 \mathrm{AU}\left(2.5^{\prime \prime}\right)$.

The first observations of the $\mathrm{p}-\mathrm{H}_{2} \mathrm{O} 2_{02}-1_{11}$, o- $\mathrm{H}_{2} \mathrm{O} 2_{21}-2_{12}$ and $\mathrm{o}-\mathrm{H}_{2} \mathrm{O} 2_{12}-1_{01}$ water lines and the rare $\mathrm{o}-\mathrm{H}_{2}^{17} \mathrm{O} 1_{10}-1_{01}$ and $\mathrm{p}-\mathrm{H}_{2}^{18} \mathrm{O} 1_{11}-0_{00}$ isotopologues towards a massive protostar are presented here.

\section{Observations and data reduction}

W3 IRS5 was observed with HIFI as part of the science demonstration phase, between March 3-22, 2010. The position observed is at RA 02:25:40.6 and Dec +62:05:51 (J2000), close to the peak radio and mid-IR emission (van der Tak et al. 2005). The observations are part of the priority science program (PSP) of the guaranteed time key program Water In Star-forming regions with Herschel (WISH; van Dishoeck et al., in prep).

Data were taken simultaneously in $\mathrm{H}$ and $\mathrm{V}$ polarizations using both the acousto-optical wide-band spectrometer (WBS) with 1.1 MHz resolution and the correlator-based highresolution spectrometer (HRS) with $480 \mathrm{kHz}$ resolution (resolutions of 0.30 and $0.13 \mathrm{~km} \mathrm{~s}^{-1}$, respectively, for the $1100 \mathrm{GHz}$ lines). We used the double beam switch observing mode with a throw of $3^{\prime}$. HIFI receivers are double sideband with a sideband ratio close to unity.

The frequencies, energy of the upper levels, system temperatures, integration times, rms noise level at a given spectral resolution, the beam size and efficiency $B_{\text {eff }}$, and the observed continuum level for each of the lines are provided in Table 1. Typical uncertainty in the frequencies is on the order of $100 \mathrm{kHz}$ (Pearson et al. 1991). Calibration of the raw data onto $T_{\mathrm{A}}$ scale was performed by the in-orbit system (Roelfsema 2010); conversion to $T_{\mathrm{mb}}$ was done with a beam efficiency estimated by raster maps of Mars (Table 1, R. Moreno, priv. comm.). The forward efficiency is 0.96. Currently, the flux scale accuracy is estimated to be between 5 and $10 \%$. Data was calibrated in the Herschel interactive processing environment (HIPE, Ott 2010) version 3.0. Further analysis was done within the CLASS ${ }^{1}$ package. After inspection, data from the two polarizations were averaged.

${ }^{1}$ http://wWw.iram.fr/IRAMFR/GILDAS

\section{Results and analysis}

Our interpretation of the spectra uses the following terms: inner and outer envelope (as defined in Sect. 1), cold molecular cloud (the region with $T \sim 10 \mathrm{~K}$ in which the protostellar envelope is embedded), and foreground cloud (unrelated molecular material in the line of sight at a different $\left.V_{\mathrm{LSR}}\right)$.

The continuum subtracted water spectra are shown in Fig. 2. For the $\mathrm{o}-\mathrm{H}_{2}^{17} \mathrm{O} 1_{10}-1_{01}, \mathrm{p}-\mathrm{H}_{2} \mathrm{O} 2_{02}-1_{11}, \mathrm{p}-\mathrm{H}_{2}^{18} \mathrm{O} 1_{11}-0_{00}$, and p$\mathrm{H}_{2} \mathrm{O} 1_{11}-\mathrm{O}_{00}$ lines, we show the HRS spectra. For the o- $\mathrm{H}_{2} \mathrm{O} 2_{21}$ $2_{12}$ and $\mathrm{o}-\mathrm{H}_{2} \mathrm{O} 2_{12}-1_{01}$ lines, we show the WBS spectra because the velocity range covered by the HRS for those lines is not sufficient. The o- $\mathrm{H}_{2} \mathrm{O} 2_{21}-2_{12}$ line is contaminated by a $\mathrm{CH}$ line $(1661.113056 \mathrm{GHz})$ seen in absorption at $\sim-58 \mathrm{~km} \mathrm{~s}^{-1}$. The $\mathrm{p}-$ $\mathrm{H}_{2} \mathrm{O} 1_{11}-0_{00}$ and $\mathrm{o}-\mathrm{H}_{2} \mathrm{O} 22_{12}-1_{01}$ lines show a narrow absorption feature at $-20.4 \mathrm{~km} \mathrm{~s}^{-1}$. Since W3 IRS5 is located very close to the galactic plane $\left(\mathrm{b} \sim 1.2^{\circ}\right)$, this water absorption is probably produced by a cold $(T \sim 10 \mathrm{~K})$ foreground cloud on the line of sight. Other cases of absorption due to clouds along the line of sight toward similar sources are reported by Marseille et al. (2010) and Wyrowski et al. (2010).

\subsection{Emission and absorption features}

Most of the water emission spectra in W3 IRS5 can be described as the sum of two velocity components: one broad $(F W H M=$ $\left.33-40 \mathrm{~km} \mathrm{~s}^{-1}\right)$ and one medium $\left(F W H M=5-10 \mathrm{~km} \mathrm{~s}^{-1}\right)$. The broad component is visible in all but the rare species and is centered approximately on the $V_{\mathrm{LSR}}$ of the source (see the broad component for the $988 \mathrm{GHz}$ line in Fig. 2). Boonman et al. (2003) observed the outflow in W3 IRS5 in CO $J=7-6$ with the JCMT and found a similar line width to what is in the water broad component. We assume that the broad component is due to the outflow.

The medium component exhibits emission in the $\mathrm{p}-\mathrm{H}_{2} \mathrm{O}$ $202-1_{11}$ and rare isotopologue lines with two peaks at -37 and $-41 \mathrm{~km} \mathrm{~s}^{-1}$ in the $\mathrm{p}-\mathrm{H}_{2} \mathrm{O} 22_{02}-1_{11}$ and $\mathrm{p}-\mathrm{H}_{2}^{18} \mathrm{O} 1_{11}-0_{00}$ spectra. A blend of emission and absorption is seen in the $\mathrm{p}-\mathrm{H}_{2} \mathrm{O} 1_{11}-0_{00}$, o$\mathrm{H}_{2} \mathrm{O} 2_{21}-2_{12}$, and o- $\mathrm{H}_{2} \mathrm{O} 2_{12}-1_{01}$ lines. The absorption in the $\mathrm{H}_{2} \mathrm{O}$ lines is blue-shifted (P-Cygni profile), suggesting an expansion of the envelope, as also detected by Benz et al. (2010) in the hydride lines of W3 IRS5. The expansion is probably powered by the multiple outflows known to exist in W3 IRS5 (Rodón et al. 2008). The $\mathrm{p}-\mathrm{H}_{2} \mathrm{O} 1_{11}-\mathrm{O}_{00}$ and $\mathrm{o}-\mathrm{H}_{2} \mathrm{O} 2_{12}-1_{01}$ lines have a double plateau in absorption at -38 and $-42 \mathrm{~km} \mathrm{~s}^{-1}$. The absorption at $-38 \mathrm{~km} \mathrm{~s}^{-1}$ is highly saturated. A simulation using RADEX (van der Tak et al. 2007) for a cold cloud with a kinetic temperature of $10 \mathrm{~K}, N_{\mathrm{H}_{2} \mathrm{O}}=3 \times 10^{13} \mathrm{~cm}^{-2}$, a line $F W H M$ of $2.8 \mathrm{~km} \mathrm{~s}^{-1}$, and $n_{\mathrm{H}_{2}}=1 \times 10^{4} \mathrm{~cm}^{-3}$ gives opacities of 4.0, 2.2, $2 \times 10^{-9}$ and $3 \times 10^{-7}$, respectively, for the p- $\mathrm{H}_{2} \mathrm{O} 1_{11}-0_{00}, \mathrm{o}-\mathrm{H}_{2} \mathrm{O} 22_{12}$ $1_{01}, \mathrm{o}-\mathrm{H}_{2} \mathrm{O} 2_{21}-2_{12}$, and $\mathrm{p}-\mathrm{H}_{2} \mathrm{O} 2_{02}-1_{11}$ species. This agrees with the detection of saturated absorption only in $\mathrm{p}-\mathrm{H}_{2} \mathrm{O} 1_{11}-0_{00}$ and o- $\mathrm{H}_{2} \mathrm{O} 2{ }_{12}-1_{01}$, as well as the absorption by a cold foreground cloud at $-20.4 \mathrm{~km} \mathrm{~s}^{-1}$ also detected only in those lines. The optical depth of the other water lines in cold regions is so low that no absorption is visible. The absorption at $-38 \mathrm{~km} \mathrm{~s}^{-1}$ presumably comes from the cold molecular cloud associated with the source.

The two-component profiles seen in emission in several of the water lines seem to be systematic features of protostellar objects, since it has been observed for low-mass Class 0 objects by Kristensen et al. (2010) and for intermediate-mass protostars by Johnstone (2010). Kristensen et al. (2010) propose that both the broad and medium components are associated with outflow shocks. In contrast to the low-mass protostars, the emission of 
Table 1. Lines of $\mathrm{H}_{2} \mathrm{O}$ observed with Herschel/HIFI in W3 IRS5.

\begin{tabular}{lccccccccc}
\hline \hline Water species & $\begin{array}{c}\text { Frequency } \\
(\mathrm{GHz})\end{array}$ & $\begin{array}{c}E_{u} \\
(\mathrm{~K})\end{array}$ & $\begin{array}{c}T_{\text {sys }} \\
(\mathrm{K})\end{array}$ & $\begin{array}{c}t_{\text {int }} \\
(\mathrm{s})\end{array}$ & $\begin{array}{c}\delta v \\
(\mathrm{kHz})\end{array}$ & $\begin{array}{c}\text { rms } \\
(\mathrm{K})\end{array}$ & $\begin{array}{c}\text { Beam } \\
\left({ }^{\prime \prime}\right)\end{array}$ & $\begin{array}{c}\eta_{\text {mb }} \\
\begin{array}{c}\text { cont. } \\
(\mathrm{K})\end{array}\end{array}$ \\
\hline $\mathrm{o}-\mathrm{H}_{2}^{17} \mathrm{O} 1_{10}-1_{01}$ & 552.020960 & 61.0 & 73 & 158 & 250 & 0.06 & 40.0 & 0.72 & 0.35 \\
$\mathrm{p}-\mathrm{H}_{2} \mathrm{O} 2_{02}-1_{11}$ & 987.926764 & 100.8 & 347 & 386 & 500 & 0.07 & 23.0 & 0.71 & 1.4 \\
$\mathrm{p}-\mathrm{H}_{2}^{18} \mathrm{O} 1_{11}-0_{00}$ & 1101.698256 & 53.4 & 351 & 601 & 500 & 0.09 & 20.0 & 0.71 & 2.15 \\
$\mathrm{p}-\mathrm{H}_{2} \mathrm{O} 1_{11}-0_{00}$ & 1113.342964 & 53.4 & 351 & 601 & 500 & 0.09 & 20.0 & 0.71 & 2.15 \\
$\mathrm{o}-\mathrm{H}_{2} \mathrm{O} 2_{21}-2_{12}$ & 1661.007637 & 194.1 & 1609 & 1164 & 250 & 0.25 & 13.5 & 0.69 & 5.5 \\
$\mathrm{o}-\mathrm{H}_{2} \mathrm{O} 2_{12}-1_{01}$ & 1669.904775 & 114.4 & 1609 & 1164 & 250 & 0.25 & 13.5 & 0.69 & 5.5 \\
\hline
\end{tabular}
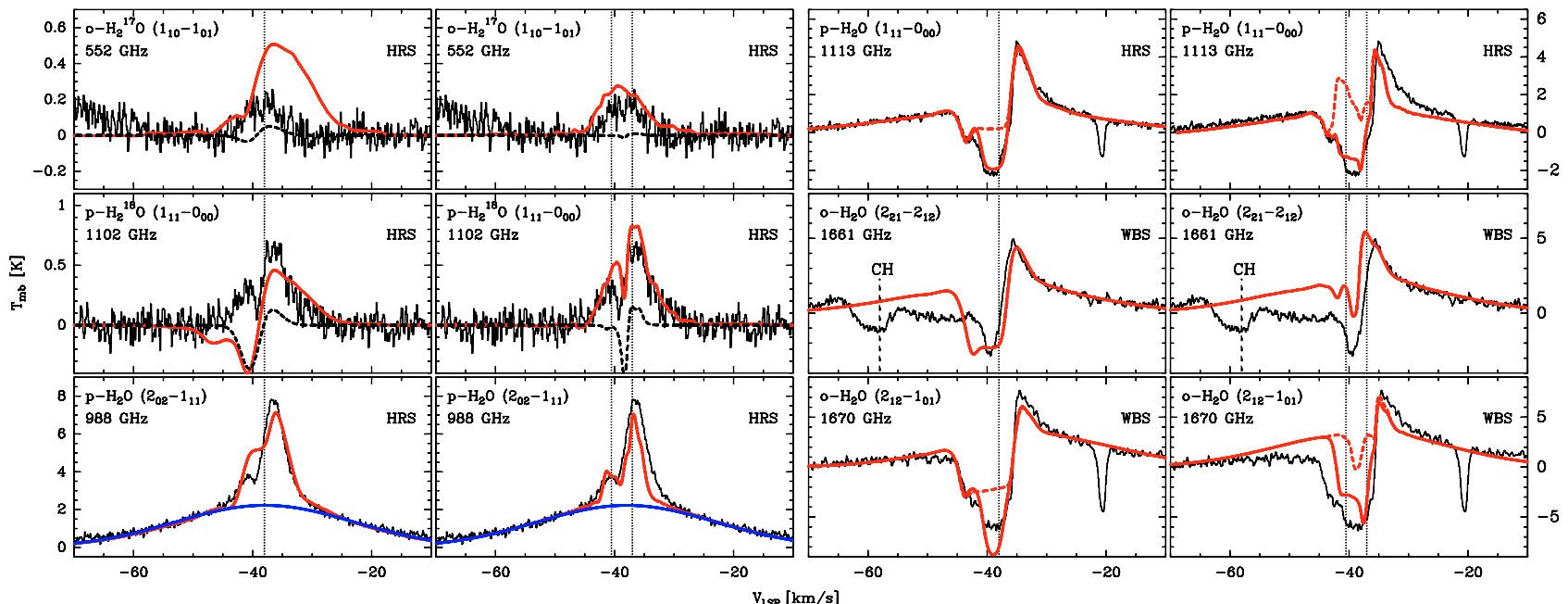

Fig. 2. HIFI spectra of water lines observed in W3 IRS5. Columns 1 and 2 show the spectra for the o- $\mathrm{H}_{2}^{17} \mathrm{O} 1_{10}-1_{01}, \mathrm{p}-\mathrm{H}_{2}^{18} \mathrm{O} 1_{11}-0_{00}$ and p- $\mathrm{H}_{2} \mathrm{O}$ $202-1_{11}$ lines, Cols. 3 and 4 show the spectra for the p- $\mathrm{H}_{2} \mathrm{O} 1_{11}-0_{00}, \mathrm{o}-\mathrm{H}_{2} \mathrm{O} 2_{21}-2_{12}$ and o- $\mathrm{H}_{2} \mathrm{O} 2_{12}-1_{01}$ lines. The single protostellar envelope model fits are shown as red lines over the spectra in Cols. 1 and 3. The two protostellar envelopes model is shown as red lines over the spectra in Cols. 2 and 4. The dashed red line over the $\mathrm{p}-\mathrm{H}_{2} \mathrm{O} 1_{11}-0_{00}$ and o- $\mathrm{H}_{2} \mathrm{O} 2_{12}-1_{01}$ spectra show the corresponding model without the cold molecular cloud (see Sect. 3.1). The dashed black line over the $\mathrm{o}-\mathrm{H}_{2}^{17} \mathrm{O} 1_{10}-1_{01}$ and $\mathrm{p}-\mathrm{H}_{2}^{18} \mathrm{O} 1_{11}-0_{00}$ spectra shows the model without the abundance jump. Vertical dotted lines indicate the model $V_{\mathrm{LSR}}$ at $-38 \mathrm{~km} \mathrm{~s}^{-1}$ for the single protostellar envelope model (Cols. 1 and 3 ) and -37 and $-40.5 \mathrm{~km} \mathrm{~s}$ for the two protostellar envelopes model (Cols. 2 and 4). Vertical dashed lines in the $\mathrm{o}-\mathrm{H}_{2} \mathrm{O} 2_{21}-2_{12}$ spectra show the position of the $\mathrm{CH}$ line. As an example, the outflow component for the p- $\mathrm{H}_{2} \mathrm{O} 2_{02}-1_{11}$ line is shown in blue. The temperature scales are shown on the left side of the figure for Cols. 1 and 2 and on the right side for Cols. 3 and 4.

the rare isotopologues only comes from the medium component and not from the broad outflow.

\subsection{Two protostellar objects seen in the optically thin lines?}

Double peaks in emission at $V_{\mathrm{LSR}} \approx-36$ and $-40 \mathrm{~km} \mathrm{~s}^{-1}$ in the mostly optically thin $\mathrm{p}-\mathrm{H}_{2} \mathrm{O} 2_{02}-1_{11}$ and $\mathrm{p}-\mathrm{H}_{2}^{18} \mathrm{O} 1_{11}-0_{00}$ line profiles suggest the detection of more than one protostellar envelope. Since the interferometric observations from Rodón et al. (2008) show that there are two millimeter sources within the HIFI beam (Fig. 1) that dominate the dust continuum emission in this region, it is possible that the two peaks correspond to emission from these two high-mass protostellar objects.

Ground-based observations of $\mathrm{C}^{17} \mathrm{O}, \mathrm{CS}, \mathrm{SO}$, and $\mathrm{CH}_{3} \mathrm{OH}$ molecules (van der Tak et al. 2003; van der Tak et al. 2006; Helmich \& van Dishoeck 1997) show lines that cover the entire velocity range of emission, but they do not help distinguish between one or two protostellar envelopes. The continuum observations by Rodón et al. (2008) could not measure the velocities of MM1 and MM2.

Even though the hypothesis of the detection of two protostellar envelopes in the spectra needs to be investigated in more detail (to be presented in a forthcoming paper), we show in Sect. 3.3 that a two protostellar envelope model tends to fit
Table 2. Parameters of single and two protostellar envelope (PE) models.

\begin{tabular}{l|r|rr}
\hline \hline Parameter & Single PE & PE 1 & PE 2 \\
\hline Outer radius (AU) & 12000 & 12000 & 12000 \\
Luminosity $\left(L_{\odot}\right)$ & $10^{5}$ & $5 \times 10^{4}$ & $5 \times 10^{4}$ \\
Mass $\left(M_{\odot}\right)$ & 250 & 125 & 125 \\
$X_{\mathrm{H}_{2} \mathrm{O}}$ & $2 \times 10^{-8}$ & $1.8 \times 10^{-8}$ & $8 \times 10^{-10}$ \\
Post-jump $X_{\mathrm{H}_{2} \mathrm{O}}$ & $1 \times 10^{-4}$ & $1 \times 10^{-4}$ & $1 \times 10^{-4}$ \\
$V_{\text {tur }}\left(\mathrm{km} \mathrm{s}^{-1}\right)$ & 2.0 & 0.4 & 0.5 \\
$V_{\exp }\left(\mathrm{km} \mathrm{s}^{-1}\right)$ & 2.0 & 1.1 & 1.2 \\
$V_{\text {LSR }}\left(\mathrm{km} \mathrm{s}^{-1}\right)$ & -38 & -37.0 & -40.5 \\
\hline
\end{tabular}

the observations better than does a single protostellar envelope model.

\subsection{A model of W3 IRS5}

We used the Monte Carlo code MC3D (Wolf 2003) and the radiative transfer program RATRAN (Hogerheijde \& van der Tak 2000) to model the water emission in W3 IRS5 following the method described in Marseille et al. (2008) with a power-law density exponent of -1.2 . The models assume spherical symmetry. A more detailed investigation of the region's morphology using 2D models is in progress and will be published when the entire set of water lines (including maps) is observed. 
Our models in W3 IRS5 have three components: an outflow, one (or two) protostellar envelopes, and a cold cloud. The outflow contribution to the spectra was modeled as a Gaussian emission line with $F W H M$ between 33 and $40 \mathrm{~km} \mathrm{~s}^{-1}$. The cold cloud was introduced taking the cloud parameters described in Sect. 3.1 for the saturated absorption. Three input parameters are used to fit the line profiles in the envelope model: water abundance $\left(X_{\mathrm{H}_{2} \mathrm{O}}\right)$, turbulent velocity $\left(V_{\mathrm{tur}}\right)$, and expansion velocity $\left(V_{\exp }\right)$. The width of the line is adjusted by changing $V_{\text {tur. }}$. The line asymmetry is reproduced by the expansion velocity $V_{\text {exp. }}$. The line intensity is fit by a combination of the abundance, $V_{\text {tur }}$, and $V_{\text {exp }}$ parameters. The same $V_{\text {tur }}$ and $V_{\text {exp }}$ have been assumed for all lines. We use abundance ratios of 500 for $\mathrm{H}_{2} \mathrm{O} / \mathrm{H}_{2}^{18} \mathrm{O}$, 4.13 for $\mathrm{H}_{2}^{18} \mathrm{O} / \mathrm{H}_{2}^{17} \mathrm{O}$ (as measured in the $\mathrm{W} 3$ region by Penzias 1981; Wilson \& Rood 1994; Wouterloot et al. 2008), and 3 for ortho/para- $\mathrm{H}_{2} \mathrm{O}$. The models assume a jump in the abundance in the inner envelope at $100 \mathrm{~K}$ with a constant inner abundance of $10^{-4}$. Having the jump occur at the radius predicted by the physical model leads to overestimating the water line intensity in the inner envelope. We thus decreased the size of the emitting region (to $\sim 1500 \mathrm{AU}$ ) by putting the abundance jump at a temperature of $150 \mathrm{~K}$. An alternative model leading to similar results keeps the jump at $100 \mathrm{~K}$ and decreases the water abundance in the inner envelope by a factor of 10 . We assume that the line intensities from the various components can simply be added as in the optically thin limit, and future models will treat this effect in more detail. Table 2 gives the parameters used in the models and the model spectra are shown in Fig. 2 for both a single and a double protostellar envelope model. The outer radius in the table corresponds to the deconvolved radius taken from the $1.3 \mathrm{~mm}$ continuum emission (Oldham et al. 1994).

The observed lines were fit using the following strategy. First, we modeled the rare isotopologue lines and then the $\mathrm{p}-\mathrm{H}_{2} \mathrm{O}$ $202-1_{11}$ line (including the outflow emission) using the same $X_{\mathrm{H}_{2} \mathrm{O}}, V_{\text {tur }}$, and $V_{\text {exp }}$ values. Once we were able to reproduce the main features of those profiles by minimizing residuals on a grid of values, we applied the same parameters to the rest of the lines, including an outflow component when justified. For the single protostellar envelope model, it was not possible to correctly fit the rare isotopologues and $\mathrm{p}-\mathrm{H}_{2} \mathrm{O} 2_{02}-1_{11}$ lines using the same $X_{\mathrm{H}_{2} \mathrm{O}}, V_{\text {tur }}$, and $V_{\text {exp }}$ values. For the double protostellar envelope model, all of the spectra are better fit with the exception of o- $\mathrm{H}_{2} \mathrm{O} 2_{21}-2_{12}$. The deep absorption in the $\mathrm{p}-\mathrm{H}_{2} \mathrm{O} 1_{11}-0_{00}$ and $\mathrm{o}-\mathrm{H}_{2} \mathrm{O} 2{ }_{12}-1_{01}$ lines is reproduced well only if we add a cold cloud to the model. The uncertainties for the water abundances are about a factor of 3 and for $V_{\text {tur }}$ and $V_{\exp }$ about $\sim 1 \mathrm{~km} \mathrm{~s}^{-1}$.

As shown in Fig. 2, the emission in the rare isotopologue lines is only reproduced in our models by including a jump in the water abundance in the inner envelope. The estimated optical depths for the lines of the rare species are low $(\tau \sim 0.1$, estimated using RATRAN). This suggests that the emission in the rare species comes from the inner envelope where the water abundance is greatly enhanced. Emission from this region could be the expected contribution from the passively (radiatively) heated inner envelope.

\section{Summary}

Spectra of six water lines observed with the Herschel/HIFI instrument in the high-mass star-forming region W3 IRS5 are presented and discussed. A 1D radiative transfer model was applied to estimate water abundances and study the kinematics in the protostellar envelope. The main results follow:

1. We detect strong water emission and absorption;

2. A strong outflow component is detected in the water lines;

3. Water emission shows absorption from cool molecular gas hosting the protostellar envelope. Blueshifted absorption suggests an expansion of the outer envelope, and no infall signature is detected;

4. Radiative transfer models indicate water abundances ranging from $10^{-8}$ to $10^{-10}$ in the outer envelope;

5. Based on our model, a jump in water abundance in the inner envelope is needed to reproduce the $\mathrm{H}_{2}^{17} \mathrm{O}$ and $\mathrm{H}_{2}^{18} \mathrm{O}$ lines.

6. The optically thin line profiles are better fit using a model with two protostellar envelopes, in agreement with previous interferometric continuum observations of W3 IRS5.

Acknowledgements. HIFI has been designed and built by a consortium of institutes and university departments from across Europe, Canada, and the United States under the leadership of SRON Netherlands Institute for Space Research, Groningen, The Netherlands, and with major contributions from Germany, France, and the US. Consortium members are: Canada: CSA, U. Waterloo; France: CESR, LAB, LERMA, IRAM; Germany: KOSMA, MPIfR, MPS; Ireland, NUI Maynooth; Italy: ASI, IFSI-INAF, Osservatorio Astrofisico di Arcetri- INAF; Netherlands: SRON, TUD; Poland: CAMK, CBK; Spain: Observatorio Astronómico Nacional (IGN), Centro de Astrobiología (CSIC-INTA). Sweden: Chalmers University of Technology - MC2, RSS \& GARD; Onsala Space Observatory; Swedish National Space Board, Stockholm University - Stockholm Observatory; Switzerland: ETH Zurich, FHNW; USA: Caltech, JPL, NHSC. HIPE is a joint development by the Herschel Science Ground Segment Consortium, consisting of ESA, the NASA Herschel Science Center, and the HIFI, PACS and SPIRE consortia. We also thank the French Space Agency CNES for financial support.

\section{References}

Benz, A. O., Bruderer, S., van Dishoeck, E. F., et al. 2010, A\&A, 521, L35 Boonman, A. M. S., Doty, S. D., van Dishoeck, E. F., et al. 2003, A\&A, 406, 937

de Graauw, Th., Helmich, F. P., Phillips, T. G., et al. 2010, A\&A, 518, L6 Doty, S. D., \& Neufeld, D. A. 1997, ApJ, 489, 122

Hachisuka, K., Brunthaler, A., Menten, K. M., et al. 2006, ApJ, 645, 337

Helmich, F. P., \& van Dishoeck, E. F. 1997, A\&AS, 124, 205

Hogerheijde, M. R., \& van der Tak, F. F. S. 2000, A\&A, 362, 697

Johnstone, D., Fich, M., McCoey, C., et al. 2010, A\&A, 521, L41

Kristensen, L. E., Visser, R., van Dishoeck, E. F., et al. 2010, A\&A, 521, L30

Ladd, E. F., Deane, J. R., Sanders, D. B., \& Wynn-Williams, C. G. 1993, ApJ, 419, 186

Marseille, M., Bontemps, S., Herpin, F., van der Tak, F. F. S., \& Purcell, C. R. 2008, A\&A, 488, 579

Marseille, M. G., van der Tak, F. F. S., Herpin, F., et al. 2010, A\&A, 521, L32

Megeath, S. T., Wilson, T. L., \& Corbin, M. R. 2005, ApJ, 622, L141

Oldham, P. G., Griffin, M. J., Richardson, K. J., \& Sandell, G. 1994, A\&A, 284, 559

Ott., S. 2010, ADASS XIX, ed. Y. Mizumoto, K.-I. Morita, \& M. Ohnishi, in press

Pearson, J. C., De Lucia, F. C., Anderson, T., Herbst, E., \& Helminger, P. 1991, ApJ, 379, L41

Penzias, A. A. 1981, ApJ, 249, 518

Pilbratt, G. L., Riedinger, J. R., Passvogel, T., et al. 2010, A\&A, 518, L1

Rodón, J. A., Beuther, H., Megeath, S. T., \& van der Tak, F. F. S. 2008, A\&A, 490, 213

Roelfsema, P. R., Helmich, F. P., Teyssier, D., et al. 2010, A\&A, submitted

van der Tak, F. F. S., Boonman, A. M. S., Braakman, R., \& van Dishoeck, E. F. 2003, A\&A, 412, 133

van der Tak, F. F. S., Tuthill, P. G., \& Danchi, W. C. 2005, A\&A, 431, 993

van der Tak, F. F. S., Walmsley, C. M., Herpin, F., \& Ceccarelli, C. 2006, A\&A, 447, 1011

van der Tak, F. F. S., Black, J. H., Schöier, F. L., Jansen, D. J., \& van Dishoeck, E. F. 2007, A\&A, 468, 627

Wilson, T. L., \& Rood, R. 1994, ARA\&A, 32, 191

Wilson, T. L., Boboltz, D. A., Gaume, R. A., \& Megeath, S. T. 2003, ApJ, 597, 434

Wolf, S. 2003, ApJ, 582, 859

Wouterloot, J. G. A., Henkel, C., Brand, J., \& Davis, G. R. 2008, A\&A, 487, 237 Wynn-Williams, C. G., Becklin, E. E., \& Neugebauer, G. 1972, in BAAS, 4, 232 Wyrowski, F., van der Tak, F. F. S., Herpin, F., et al. 2010, A\&A, 521, L34 Zinnecker, H., \& Yorke, H. W. 2007, ARA\&A, 45, 481 
1 Université de Bordeaux, Laboratoire d'Astrophysique de Bordeaux, France; CNRS/INSU, UMR 5804, Floirac, France

e-mail: luisagustinchavarria@gmail.com

2 SRON Netherlands Institute for Space Research, PO Box 800, 9700 $\mathrm{AV}$, Groningen, The Netherlands

3 Kapteyn Astronomical Institute, University of Groningen, PO Box 800, $9700 \mathrm{AV}$, Groningen, The Netherlands

${ }^{4}$ École des Mines de Nantes, 4 rue Alfred Kastler, 44300 Nantes, France

5 Max-Planck-Institut für Radioastronomie, Auf dem Hügel 69, 53121 Bonn, Germany

${ }^{6}$ Leiden Observatory, Leiden University, PO Box 9513, 2300 RA Leiden, The Netherlands

7 Max Planck Institut für Extraterrestrische Physik, Giessenbachstrasse 1, 85748 Garching, Germany

8 Centro de Astrobiología. Departamento de Astrofísica. CSIC-INTA. Carretera de Ajalvir, Km 4, Torrejón de Ardoz, 28850 Madrid, Spain

9 Observatorio Astronómico Nacional (IGN), Calle Alfonso XII,3. 28014 Madrid, Spain

${ }^{10}$ INAF - Istituto di Fisica dello Spazio Interplanetario, Area di Ricerca di Tor Vergata, via Fosso del Cavaliere 100, 00133 Roma, Italy

11 Institute of Astronomy, ETH Zurich, 8093 Zurich, Switzerland

12 Department of Astronomy, The University of Michigan, 500 Church Street, Ann Arbor, MI 48109-1042, USA

13 Department of Radio and Space Science, Chalmers University of Technology, Onsala Space Observatory, 43992 Onsala, Sweden

14 California Institute of Technology, Division of Geological and Planetary Sciences, MS 150-21, Pasadena, CA 91125, USA

15 School of Physics and Astronomy, University of Leeds, Leeds LS2 9JT, UK

16 INAF - Osservatorio Astrofisico di Arcetri, Largo E. Fermi 5, 50125 Firenze, Italy

17 Astronomical Institute Anton Pannekoek, University of Amsterdam, Kruislaan 403, 1098 SJ Amsterdam, The Netherlands

18 Department of Astrophysics/IMAPP, Radboud University Nijmegen, PO Box 9010, 6500 GL Nijmegen, The Netherlands
19 Department of Physics and Astronomy, Denison University, Granville, OH 43023, USA

${ }^{20}$ LERMA and UMR 8112 du CNRS, Observatoire de Paris, 61 Av. de l'Observatoire, 75014 Paris, France

21 University of Waterloo, Department of Physics and Astronomy, Waterloo, Ontario, Canada

22 Observatorio Astronómico Nacional, Apartado 112, 28803 Alcalá de Henares, Spain

23 INAF - Osservatorio Astronomico di Roma, 00040 Monte Porzio catone, Italy

24 MPI für Sonnensystemforschung, 37191 Katlenburg-Lindau, Germany

25 National Research Council Canada, Herzberg Institute of Astrophysics, 5071 West Saanich Road, Victoria, BC V9E 2E7, Canada

26 Department of Physics and Astronomy, University of Victoria, Victoria, BC V8P 1A1, Canada

27 Centre for Star and Planet Formation, Natural History Museum of Denmark, University of Copenhagen, Øster Voldgade 5-7, 1350 Copenhagen K., Denmark

28 Department of Astronomy, Stockholm University, AlbaNova, 106 91 Stockholm, Sweden

29 California Institute of Technology, Cahill Center for Astronomy and Astrophysics, MS 301-17, Pasadena, CA 91125, USA

30 The University of Western Ontario, Department of Physics and Astronomy, London, Ontario, N6A 3K7, Canada

31 Harvard-Smithsonian Center for Astrophysics, 60 Garden Street, MS 42, Cambridge, MA 02138, USA

32 Jet Propulsion Laboratory, California Institute of Technology, Pasadena, CA 91109, USA

33 Department of Physics and Astronomy, University of Calgary, Calgary, T2N 1N4, AB, Canada

34 Instituto de Radioastronomía Milimétrica (IRAM), Avenida Divina Pastora 7, Núcleo Central, 18012 Granada, Spain

35 KOSMA, I. Physik. Institut, Universität zu Köln, Zülpicher Str. 77, 50937 Köln, Germany

36 N. Copernicus Astronomical Center, Rabianska 8, 87-100, Torun, Poland 